\title{
Exámenes telemáticos: aprendizaje y calificación. Problemas técnicos y jurídicos
}

\author{
José Antonio Tardío Pato \\ Profesor titular de Derecho Administrativo de la \\ Universidad Miguel Hernández de Elche (Alicante, España) \\ j.a.tardio@umh.es | https://orcid.org/0000-0001-8195-2123
}

\section{Extracto}

Este trabajo constituye un análisis de los exámenes telemáticos en su doble vertiente: de instrumento de aprendizaje, por un lado, y de mecanismo calificador, por otro. Se efectúa un estudio de los exámenes telemáticos en las ciencias jurídicas tanto desde su perspectiva técnica como jurídica y así, se examina, desde un punto de vista jurídico, el uso de la cámara de control en la realización de dichos exámenes y en la grabación de los mismos, así como de los demás medios de vigilancia informática (e-proctoring). Se llega a la conclusión de la necesidad de ponderación (propia del principio de proporcionalidad) de determinados derechos del estudiante tomado como referencia, por un lado, y de la eventual lesión, por dicho estudiante, de determinadas normas y de ciertos derechos del resto del alumnado, por otro. Como derechos que hay que ponderar del estudiante de referencia, cabe destacar los derechos a la propia imagen, a la intimidad, a la inviolabilidad del domicilio y a la protección de datos. Y, en la otra parte de la balanza, hay que resaltar, entre otros bienes jurídicos protegidos, la posible lesión del derecho del resto del alumnado a la igualdad de oportunidades y no discriminación en el ejercicio de sus derechos académicos y la vulneración del derecho a la evaluación del rendimiento académico con criterios públicos y objetivos, que, claramente, excluye la evaluación con fraude.

Palabras clave: exámenes universitarios; fraude en exámenes; vigilancia de exámenes telemáticos; evaluación; calificación; protección de datos; igualdad de oportunidades y no discriminación. 


\title{
Online exams: learning and grading. Technical and legal problems
}

\author{
José Antonio Tardío Pato
}

\section{Abstract}

This work constitutes an analysis of the online exams in their double aspect, as a learning instrument, on the one hand, and a qualifying mechanism, on the other. A study of the online examinations in legal sciences is carried out both from their technical and legal perspective and thus, from a legal point of view, is examined the use of the video camera to monitor the aforementioned exams and record them, as well as of the other means of computer surveillance (e-proctoring). And the conclusion is the need for weighting up (in line with the principle of proportionality) certain rights of the student taken as a reference, on the one hand, and the possible infringement by the student of certain rules and certain rights of other students, on the other. As rights from the reference student to be weighted up, it should be highlighted the rights to self-image, privacy, inviolability of the home and data protection. And, on the other side of the scale, we must highlight, among other protected legal rights, the possible injury to the right of other students to equal opportunities and non-discrimination in the exercise of their academic rights and the violation of the right to the evaluation of academic performance with public and objective criteria, which clearly excludes the evaluation with fraud.

Keywords: university exams; exam fraud; proctoring of online exams; evaluation; grading; data protection; equal opportunities and non-discrimination.

Citation: Tardío Pato, J. A. (2021). Online exams: learning and grading. Technical and legal problems. Tecnología, Ciencia y Educación, 20, 155-176. https://doi.org/10.51302/tce.2021.598 


\section{Sumario}

1. Introducción

2. La doble función de los exámenes académicos (como instrumento de aprendizaje y como medio de evaluación del rendimiento) y las modalidades más usadas en las ciencias jurídicas

2.1. Exámenes académicos como instrumento de aprendizaje

2.2. Exámenes académicos como medio de evaluación del rendimiento

3. La elección de la modalidad de exámenes telemáticos, atendiendo a su vertiente de medio de aprendizaje y a su vertiente calificadora, con la doble exigencia de valoración lo más justa y veraz posible que evite efectos fraudulentos

4. La especial problemática jurídica del uso de cámara de control en la realización de los exámenes telemáticos y de la grabación de dichos exámenes, así como de los demás medios de vigilancia informática (e-proctoring)

4.1. Problemática jurídica del uso de cámara de control en la realización de los exámenes telemáticos

4.2. Problemática jurídica de la grabación de exámenes telemáticos

4.3. Tratamiento de los datos personales

4.4. Reglamentos de necesidad

4.5. Conclusiones

Referencias bibliográficas

Nota: la base del presente artículo se halla en la ponencia expuesta en el II Congreso Internacional sobre la Innovación de la Docencia Universitaria ante la Revolución de los Medios Digitales: Nuevos Retos Educativos tras el COVID-19, desde el Derecho, celebrado entre los días 16 y 19 de noviembre de 2020 en formato virtual por Blackboard Collaborate Ultra y organizado desde las sedes de la Universitat de València y de la Universidad de Málaga. 


\section{Introducción}

La docencia y los medios de evaluación telemáticos, que ya existían en los centros de enseñanza a distancia como algo característico de los mismos, se han convertido -en esta época de pandemia, con confinamientos imperativos y limitaciones de aforo en las aulasen una necesidad generalizada para todo tipo de centros educativos.

Afortunadamente, la ciencia informática y de las telecomunicaciones nos ofrecen diferentes plataformas telemáticas, con diversas aplicaciones, que permiten efectuar la citada docencia y realizar la correspondiente evaluación del aprendizaje. Pero su generalización como consecuencia del estado de necesidad ha puesto en la palestra los distintos problemas que suscitan.

Así, se invoca que los exámenes pueden sufrir problemas técnicos y, sobre todo, que se facilita el uso de mecanismos fraudulentos al estudiante, como la copia de textos en papel, de archivos procedentes de internet, de teléfonos móviles o de otros dispositivos informáticos que se ocultan al docente; el estudiante al que se examina puede ser suplantado por otro; el examinando puede recibir «soplos» de terceros situados en las inmediaciones, sin ser vistos, o a través de WhatsApp, de aplicaciones similares, mediante pinganillos o en virtud de conexiones a su ordenador por control remoto, etc ${ }^{1}$.

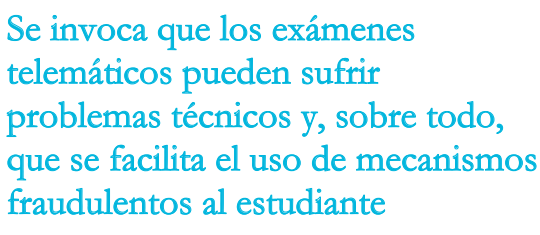

Se invoca que los exámenes telemáticos pueden sufrir problemas técnicos y, sobre todo, que se facilita el uso de mecanismos fraudulentos al estudiante

Pero, igualmente, hay mecanismos tecnológicos que posibilitan contrarrestar tal fraude ${ }^{2}$, como, por ejemplo, el uso por parte de los docentes de videocámaras para comprobar cómo realiza el examinado su examen ${ }^{3}$ y el empleo de los denominados «servicios de vigilancia de

1 Se habla de «riesgos de fraude» (riesgos confiabilidad) y de «riesgos de fallos telemáticos» (riesgos técnicos) y se subraya que el docente debe saber gestionarlos con las oportunas contramedidas, condicionadas por los recursos tecnológicos y humanos, que suponen necesariamente un coste en tiempo o recursos. Hay que buscar el equilibrio entre coste y beneficio (véase Estepa et al., 2020, pp. 1-3).

2 En Estepa et al. (2020), aparte de describirse y recomendarse medidas para la mitigación del riesgo técnico, tales como el ajuste de la red local, la reducción de carga, etc. (ibidem, pp. 4-5), se nos indican algunas de las contramedidas para mitigar el llamado «riesgo confiabilidad», como activación del audiovídeo durante el examen, el control de presencia automatizado ante la cámara, etc. (ibidem, pp. 6 y ss.).

3 El mecanismo que aconsejó el correspondiente vicerrectorado de la Universidad Miguel Hernández en unas instrucciones sobre la realización de exámenes de 13 de mayo de 2020, para la fase de confinamiento derivado de la situación de pandemia, fue el de la utilización de dos plataformas informáticas distintas: una 
exámenes en línea» (servicios e-proctoring o programas online proctored exams), para la identificación del alumnado y para vigilar automáticamente su entorno visual y auditivo e impedir que puedan conectarse con otros dispositivos o que abran pestañas diferentes a la propia del examen ${ }^{4}$.

Entre ellos, cabe citar Safe Exam Browser ${ }^{5}$ (navegador seguro para exámenes) y Respondus LockDown Browser ${ }^{6}$, por estar usándose actualmente en universidades españolas.

\section{Determinados sectores del alumnado se han alzado para invocar la protección de derechos que, eventualmente, pudieran ser vulnerados cuando se realizan exámenes usando medios telemáticos (derechos a la intimidad, a la propia imagen, a la inviolabilidad del domicilio o a la protección de datos)}

Sin embargo, inmediatamente, determinados sectores del alumnado se han alzado para invocar la protección de derechos que, eventualmente, pudieran ser vulnerados cuando se realizan exámenes usando medios tele-

para la realización del examen en sí y otra para la vigilancia del examen con cámaras. Esta última consistió en que cada estudiante enfocara con la cámara de sus móviles personales, en modo selfi, la pantalla y el teclado de su propio ordenador, con el cual estaba efectuando el examen, y también la mesa o escritorio en el que estaban situados la pantalla y el teclado.

En la Guía de Pruebas de Evaluación en Tiempos de Docencia Online por Emergencia (Universidad Carlos III de Madrid, 2020) se dice que «las pruebas de evaluación síncrona tienen que combinarse con vigilancia por parte del profesorado utilizando Google Meet y solicitando a los estudiantes que activen su cámara web. Los estudiantes que no tengan cámara web en su equipo informático deben conectarse mediante el teléfono móvil» (p. 11).

La prueba de la existencia de cierta semejanza entre los mecanismos garantizadores de la veracidad del resultado utilizados en otros países y los empleados en España la podemos observar en el trabajo de Servadio et al. (2020, p. 165, en especial p. 167), que describen los usados en Italia en determinados centros.

4 El informe del gabinete del ministro de Universidades de España, redactado por González et al. (2020, pp. 13 y ss.), nos ofrece una enumeración de los mismos.

5 Puede consultarse en el enlace https://moodle.com/es/news/moodle-and-safe-exam-browser/\#: :text= \% C2\%BFQu\%C3\%A9\%20es\%20el\%20navegador\%20de,realizan\%20una\%20evaluaci\%C3\% B3n\%20en\%20I\%C3\%ADnea. Safe Exam Browser es descrito como «un navegador de bloqueo de código abierto» (adquirido de manera gratuita) que permite a los docentes restringir el acceso de los estudiantes a sitios web externos, a funciones del sistema o a otras aplicaciones mientras realizan una evaluación en línea. Y se destaca como un mecanismo que, junto a la supervisión en persona (la visión personal del examinado y su identificación), sirve para garantizar la honestidad de la evaluación.

6 En la guía de la Universidad Carlos III de Madrid (2020) se indica que «Respondus LockDown Browser es una herramienta de e-proctoring que permite aumentar la integridad de la realización de exámenes (cuestionarios) a través del Aula Global (Moodle) por parte de los estudiantes mediante el control/bloqueo de las aplicaciones del ordenador que el estudiante puede utilizar simultáneamente. Además de la configuración que debe hacerse en una sección del curso en Aula Global (Moodle), será necesario que el estudiante se descargue un navegador web especial, que será el que controle que el alumno no accede ni utiliza información y [un] software no permitido» (pp. 48 y ss.). También se nos da noticias de Respondus LockDown Browser en González et al. (2020, pp. 14-15). 
máticos (derechos a la intimidad, a la propia imagen, a la inviolabilidad del domicilio o a la protección de datos $)^{7}$. Sobre todo, se ha puesto énfasis especialmente en la colisión entre dichos derechos y las medidas técnicas dirigidas a garantizar la veracidad en la realización de los exámenes y en la elusión de los mencionados fraudes, claramente para dar la mayor impunidad posible a los mecanismos fraudulentos, pues el resultado al que se llega no es otro que el de hacer exámenes telemáticos sin vigilancia efectiva y sin garantías de que no ha habido fraude alguno en su ejecución.

Se olvida así, totalmente, la salvaguardia del cumplimiento de los deberes de esos estudiantes (los que cometen fraude), proclamados por nuestro ordenamiento jurídico, y se rehúye la garantía de los derechos de aquella parte del alumnado que realiza sus exámenes sin fraude y a la que se deja en desventaja comparativa.

Igualmente, parece obviarse el hecho de que, en relación con el derecho fundamental a la protección de datos, y en el caso que nos ocupa, inciden motivos de licitud del tratamiento de los correspondientes datos, como la necesidad de mecanismos antifraude «para el cumplimiento
Se olvida así, totalmente,
la salvaguardia del cumplimiento de los deberes de esos estudiantes (los que cometen fraude), proclamados por nuestro ordenamiento jurídico, y se rehúye la garantía de los derechos de aquella parte del alumnado que realiza sus exámenes sin fraude y a la que se deja en desventaja comparativa de una misión realizada en interés público o en el ejercicio de poderes públicos conferidos al responsable del tratamiento", habida cuenta de que la educación superior está calificada como «servicio público» en el artículo 1.1 de la Ley orgánica 6/2001, de 21 de diciembre, de Universidades (LOU).

Sobre estos deberes del alumnado, así como sobre los derechos de los estudiantes perjudicados por el fraude de otros y la misión de interés público, volveremos al final de nuestra exposición.

La falta de un adecuado tratamiento de dichas cuestiones -a mi entender-, que son de carácter netamente jurídico, no solo ha supuesto un perjuicio a la hora de usar las medidas técnicas frente al fraude, sino que también ha favorecido la citada impunidad del mismo y ha generado cierta animadversión a los exámenes telemáticos, en favor de la realización, a ultranza, de exámenes presenciales.

7 Sobre estas cuestiones incide el informe emitido para la Conferencia de Rectores de las Universidades Españolas (CRUE) (Martínez et al., 2020, in totum), que desde el comienzo descarta las técnicas de proctoring (vigilancia) porque pueden «plantear particulares dificultades o riesgos en materia de proporcionalidad, predeterminación normativa y necesidad de desarrollar una evaluación de impacto relativa a la protección de datos» (ibidem, p. 1). 
Y esto ha ocurrido, incluso, en el caso de aquellos docentes y autoridades académicas menos celosos de la veracidad y pulcritud de las calificaciones, en momentos de altas cotas de contagio por la COVID-19, cuando la posibilidad de realizar exámenes presenciales suponía un riesgo patente para la salud y hacer uso de la evaluación telemática fue considerada como la mejor opción desde el punto de vista de la prudencia y de los principios jurídicos de prevención y precaución (o cautela) que operan, especialmente, en los ámbitos de la salud y el medioambiente ${ }^{8}$.

\section{La doble función de los exámenes académicos (como ins- trumento de aprendizaje y como medio de evaluación del rendimiento) y las modalidades más usadas en las ciencias jurídicas}

\subsection{Exámenes académicos como instrumento de aprendizaje}

La función de los exámenes como instrumento de aprendizaje parece en desuso en la época actual, donde prevalece el fenómeno calificado como «ansia de títulos». Hemos construido un sistema social demasiado formalista en el que, la mayor parte de las veces, se da bastante más importancia al número de títulos conseguidos por los estudiantes, especialmente de másteres y similares, que al nivel de conocimiento real obtenido, a la especialización y a la adquisición de las correspondientes habilidades intelectuales.

\section{La función de los exámenes como instrumento de aprendizaje parece en desuso en la época actual, donde prevalece el fenómeno calificado como «ansia de títulos»}

8 Hay que tener presente, en este punto, que el artículo 168 del Tratado de Funcionamiento de la Unión Europea (TFUE) (versión consolidada) proclama que la acción de «la Unión [...] se encaminará a mejorar la salud pública, prevenir las enfermedades humanas y evitar las fuentes de peligro»y, en relación con ello, el artículo 191.2 del TFUE acoge los principios de precaución y prevención: «La política de la Unión en el ámbito del medioambiente [...] se basará en los principios de cautela y acción preventiva [...]”. En ambos casos, se trata de adoptar medidas adecuadas para eliminar, reducir o mitigar los efectos negativos para el medioambiente (y la salud humana), basándose en los conocimientos científico-técnicos. Pero mientras que la «prevención" supone que dichos conocimientos permiten deducir con certeza los efectos que puedan producirse y las medidas que deben adoptarse, la «precaución» implica que tales conocimientos no gozan de dicha certeza a la hora de deducir tales efectos y medidas (Kramer, 1999, p. 93).

Y, sobre la legitimidad y licitud de invocar dicho principio para la aprobación de las correspondientes medidas, incluidas las de carácter restrictivo, son muy claras varias Sentencias del Tribunal de Justicia de la Unión Europea: National Farmers Union, de 5 de mayo de 1998, asunto C-157/1996; asunto Vacas Locas, del mismo día (C-180/1996), en sus puntos 60, 99, 100,103 y 111; y asunto Afton Chemical Limited, de 8 de julio de 2010 (C-343/09). 
Frente a ello, hay que recordar que los exámenes constituyen un elemento de autoevaluación del propio estudiante, gracias al cual se comprueba cuál es realmente su nivel de conocimiento de una materia y su adquisición de habilidades intelectuales características de la misma, para verificar cuáles han sido sus errores y deficiencias; de modo que sirve para corregir tales equivocaciones y actúa como medio de retroalimentación en su aprendizaje. Así pues, es una pieza importante en el sistema de enseñanza, difícil de sustituir?.

Pero, además, en la misma vertiente, cumple, asimismo, otra función real, consistente en que la existencia de exámenes y su proximidad conduce al ser humano a esforzarse para aumentar sus conocimientos de las materias sobre las que versa el examen. Por un lado, por pundonor personal, pues a la mayor parte de las personas nos gusta obtener buenos resultados reales en una evaluación de nuestros conocimientos ${ }^{10}$. Y, por otro lado, porque, al estar próximo el examen, el ser humano se ve obligado a acelerar el aprendizaje relativo a la materia en cuestión, por las diversas consecuencias del resultado de tal examen (aprovechamiento del dinero de la matrícula, obtención de los créditos académicos que el examen puede suponer, etc.). Como regla general, pues, los exámenes impulsan la velocidad del aprendizaje.

9 Los especialistas en didáctica distinguen los conceptos de «evaluación», por un lado, y de «calificación» y «medición», por otro. Se ha dicho que «evaluar» supone, de algún modo, medir y calificar, pero no termina en ello, sino que va más allá, alcanzando otras dimensiones (Castillo y Cabrerizo, 2009, pp. 24-25). Se recuerda que «calificar», según la Real Academia Española (RAE), es «juzgar el grado de suficiencia o la insuficiencia de los conocimientos demostrados por un alumno u opositor en un examen o ejercicio"». Se destaca que la «medición» es la constatación, de forma cuantitativa, de la cantidad de un elemento y busca la objetividad (López, 2016, p. 199). La «evaluación», por su parte, es un enjuiciamiento de determinada información sobre el proceso de la enseñanza, al inicio, durante la misma y al final de esta, así como un mecanismo para comprobar en qué medida se han alcanzado los objetivos previstos (ibidem, pp. 200-201), por lo que constituye un aspecto sustantivo en el proceso de enseñanza-aprendizaje, con un sentido formativo, que posee potencialidad para orientar los aprendizajes (ibidem, p. 197), para generar una orientación a la mejora de los mismos (ibidem, p. 218). Se subraya que, "desde el enfoque de la autoevaluación [...] facilita que el estudiante tome conciencia de su situación formativa, estimulando los procesos de metacognición» y «el profesorado orienta y apoya el proceso de autoanálisis y reflexión singular de cada estudiante» (ibidem, p. 203). Aunque, en este punto, se resalta que, mientras que hay estudiantes que "actúan de un modo honesto, reflexivo y crítico», hay otros que «no respetan y asumen los principios de la plena autoevaluación» y «no asumen un proceso de autorreflexión y diagnóstico», de modo que «terminan engañándose a sí mismos, generando un clima de aula poco empático y equilibrado» (ibidem, pp. 203-204). Y, en este sentido, se culmina lo anterior destacando que la evaluación constituye un instrumento poderoso para que el estudiante aprenda a evaluar y a entender cuál es su aprendizaje individual y, de este modo, desarrolle una de las habilidades clave de «aprender a aprender», la llamada «metacognición» (Bordas y Cabrera, 2001, pp. 28 y ss).

10 Este factor psicológico ya lo destacó el filósofo y pedagogo Comenius en su Didáctica magna (cuya primera edición apareció en el año de 1630) cuando, al referirse a los exámenes, planteó la siguiente reflexión: "¿Cómo no esperaremos que han de procurar toda diligencia y esfuerzo los que saben que han de sufrir examen tan público, serio y severo?» (p. 217 de la trad. española). 
Dicho lo anterior, en esta vertiente de aprendizaje de los exámenes académicos se plantea cuál es la modalidad de examen, dentro de los más utilizados en las ciencias jurídicas, que mejor ayuda a adquirir los conocimientos necesarios para la titulación: exámenes que contengan preguntas que coincidan exactamente con los epígrafes y subepígrafes de los temas impartidos; preguntas tipo test; preguntas cortas sobre cuestiones esenciales; preguntas que exigen la interrelación de los conocimientos adquiridos; ejercicios de composición, en los que se han de desarrollar temas de cierta dimensión que no coinciden totalmente con ningún epígrafe o subepígrafe concreto de los contenidos del programa, pero que están relacionados con varios; la respuesta a casos prácticos planteados; o la realización de trabajos de resumen del estado de la cuestión en la literatura científica de la materia correspondiente y las conclusiones personales finales. A ello dedicaremos un epígrafe posterior específico de este trabajo, para sus manifestaciones telemáticas.

\subsection{Exámenes académicos como medio de evaluación del ren- dimiento}

Ahora bien, por otro lado, la vertiente más ostensible de los exámenes es la de constituir el medio, por antonomasia, de evaluación del rendimiento académico para la expedición del correspondiente título (vertiente o función calificadora), con efectos, en mayor o menor medida, oficiales ${ }^{11}$.

Aquí, lo importante es que la evaluación sea, por un lado, lo más justa posible, es decir, que mida, del mejor modo, el rendimiento académico del estudiante. Al referirnos a los diversos mecanismos evaluativos, ya indicaremos cuál nos parece más justo y equilibrado.

Y, por otro lado, debe ser lo más veraz y objetiva, de manera que no incidan mecanismos fraudulentos de ningún tipo que la falseen. En este sentido, cualquier universidad debería poner los medios para evitar el uso de tales medios fraudulentos, que son cada vez más sofisticados, tecnológicamente hablando, e incidir, especialmente, en los medios preventivos, aunque sin renunciar tampoco a las sanciones administrativas; haciendo más hincapié, en este caso, en la función disuasoria que en la punitiva que los mismos cumplen.

En relación con los exámenes presenciales, dentro de los mecanismos preventivos, como parece ser que los inhibidores de frecuencia plantean muchos problemas, cabe destacar,

11 Sobre el régimen jurídico de las calificaciones académicas han incidido los trabajos de Tardío (1986, pp. 209 y ss.; 1996, in totum) y Moreu (2003, in totum). 
por ejemplo, el que describe, en internet, la Universidad de Murcia, consistente en invadir con música las frecuencias usadas para la transmisión de voz, como, por ejemplo, la frecuencia de Bluetooth, con la finalidad de evitar la transmisión al exterior del contenido de los exámenes mediante pinganillos en los oídos ${ }^{12}$. Y noticias de prensa nos ilustran de que la misma universidad usaría en la Evaluación del Bachillerato para el Acceso a la Universidad (EBAU) rastreadores de frecuencias en las aulas, en cualquier momento, con el fin de detectar dispositivos electrónicos ${ }^{13}$. Como también nos informan de que alguna Administración no universitaria ha utilizado, para los exámenes de procesos selectivos, dispositivos que detectan en las aulas la transmisión de voz y de datos y que, incluso, permiten oír lo que se está transmitiendo desde el exterior o registrar los datos emitidos ${ }^{14}$.

\section{La elección de la modalidad de exámenes telemáticos, atendiendo a su vertiente de medio de aprendizaje y a su vertiente calificadora, con la doble exigencia de valoración lo más justa y veraz posible que evite efectos fraudulentos}

Los mecanismos de docencia telemática no han planteado especiales problemas aplicativos. El mayor problema lo ha suscitado la elección de la modalidad de examen y su desarrollo para lograr que estas pruebas cumplan con su función de actuar como instrumento de aprendizaje y conseguir, simultáneamente, que sean un buen mecanismo de evaluación del rendimiento académico, en el que incidan, en la menor medida posible, eventuales medios fraudulentos que desvirtúen su esencia y corrompan el sistema. Repasemos las principales modalidades de examen, desde ambas perspectivas, pero en su uso telemático:

\section{A) Examen tipo test}

Este tipo de examen encaja muy bien con la docencia telemática, pues no suele ser el que mejor favorece los medios fraudulentos más típicos, basados en copiar y pegar trozos de archivos. Requiere bastante trabajo del profesor, para establecer preguntas con respuestas válidas e inválidas, pero se corrigen muy fácilmente, dado que diversas aplicaciones informáticas permiten que dicha corrección pueda ser totalmente automática. Por eso,

12 https://www.youtube.com/watch?v=kbyLB3XpFng

13 Consúltese La Verdad de Murcia del 23 de enero de 2020: https://www.laverdad.es/murcia/alumnospresenten-selectividad-20200122135952-nt.html?ref=https:\%2F\%2Fwww.google.com\%2F

14 Consúltese la nota de prensa del Gobierno de Navarra de 27 de abril de 2018: https://www.navarra.es/NR/ rdonlyres/E783F9FD-A42E-4BBB-AFFC-27B4A4AF98EC/414149/descubiertoopositorcopiandonavarrahaciendapoliciaf.pdf 
ha sido quizá el tipo más utilizado por el profesorado en la etapa del primer confinamiento impuesto por la declaración del estado de alarma en todo el ámbito estatal español. Sin embargo, plantea los problemas de todo examen tipo test:

- En la realidad a la que hay que aplicar los conocimientos adquiridos, nadie nos suele dar las respuestas alternativas para elegir una, sino que tales respuestas debe buscarlas uno mismo, desde cero, la mayor parte de las veces.

- Conlleva una vertiente aleatoria muy importante. Esto implica que algunos estudiantes, que aprueban y obtienen con esta modalidad buenas calificaciones, no suelen conseguir los mismos resultados con otras modalidades de examen y no poseen profundos conocimientos de la materia; mientras que, por el contrario, hay estudiantes muy buenos, que suelen obtener calificaciones excelentes con otras modalidades de examen y que tienen profundos conocimientos de la materia, pero a los que las distintas respuestas alternativas del examen tipo test les despistan y confunden, logrando calificaciones peores.

En cualquier caso, la garantía de la veracidad del resultado requiere evitar que el examen pueda ser ejecutado por personas distintas al estudiante que se está examinando -in situ o por control remoto de su dispositivo (tipo Team Viewer o similar)-, que este abra pestañas de archivos propios o localizados en tal momento en internet o que conecte con otros dispositivos externos, lo que exige control, mediante cámaras, de la pantalla del dispositivo del estudiante, de su teclado y del puesto de realización del examen, así como de la acreditación de la identidad del estudiante a través de la exhibición previa del documento identificativo.

\section{B) Examen de preguntas en positivo}

Este tipo de exámenes se suele calificar, en la terminología de las aplicaciones al uso, como «tipo ensayo». Así se denomina, por ejemplo, en la plataforma Moodle. Con contenidos específicos, expresados en subepígrafes o párrafos concretos de un texto, son fáciles de generar, pero se tarda un tiempo importante en su corrección.

Este tipo de exámenes, si no se emplean medios informáticos o telemáticos para impedirlo, permiten cortar y pegar los textos de las respuestas desde otros archivos, con mayor o menor discreción, mediante la modificación de los correspondientes conectores lingüísticos o de determinados términos del texto copiado.

Frente a ello, sobresalen, como medios antifraude, los que impiden abrir archivos en la pantalla del dispositivo donde se está realizando el examen o imposibilitan la conexión de dicho dispositivo con otros; unido a mecanismos de control de movimientos de los ojos hacia otro dispositivo o fuente de información (tipo e-proctoring). 
Y, a lo anterior, se pueden adicionar cámaras que permitan visualizar la pantalla del dispositivo del estudiante, su teclado y el puesto de realización del examen, a la vez que acreditar la identidad del estudiante mediante la exhibición previa del documento identificativo del mismo.

Desde el punto de vista de su justicia, si se evitan fraudes, como vimos anteriormente, es el tipo de examen que admite mayor graduación en las calificaciones y, por tanto, unas notas más ajustadas a los conocimientos acreditados por cada uno.

Esta misma modalidad de examen puede realizarse de modo oral, mediante videoconferencia, y, en tal caso, el fraude resulta un poco más difícil (especialmente, copiar y pegar texto), pero no lo excluye totalmente (por ejemplo, es posible que el estudiante pueda disponer de un segundo monitor, situado a distinta altura que aquel por el que se efectúa la videoconferencia, para leer el texto).

Por otro lado, como lo aconsejable es cambiar de preguntas de un estudiante a otro, su preparación y realización lleva mucho tiempo, lo que lo hace desaconsejable a partir de determinado número de alumnos.

Y, desde luego, se impone su grabación ${ }^{15}$, como medio de prueba, especialmente en caso de revisión y de reclamación de la calificación.

\section{C) Evaluación basada en la elaboración de trabajos sobre el estado de la cuestión en la literatura científica relativos a determinados apar- tados del temario}

Este tipo de evaluación no plantea problemas adicionales a los ya examinados anteriormente para la docencia de tipo presencial.

Lo positivo de este método es que obliga al estudiante a buscar por sí mismo los mejores contenidos sobre la materia y la mejor forma de asimilarlos y explicárselos a los demás. En su variante oral, promueve ejercitar la presentación en público de los contenidos de cada asignatura.

En cuanto a los aspectos negativos, estos trabajos solo cubren ámbitos reducidos del contenido total de los temarios de las asignaturas y es fácil copiar y pegar archivos, tanto en la enseñanza presencial como en la telemática, lo que hace que, en nuestra opinión, no sea la mejor modalidad de examen, ya sea en su vertiente de medio de aprendizaje, ya sea en su faceta de medio de calificación.

15 Véase la guía de la Universidad Carlos III de Madrid (2020, p. 8). 


\section{D) Examen basado en la resolución de casos prácticos}

En modo telemático, este tipo de exámenes, al ser más difícil el fraude porque no facilitan copiar y pegar archivos, no plantean especiales problemas de veracidad de los resultados, pero sí requieren controlar que el examen no haya sido realizado por terceras personas, para lo cual resulta necesario el uso de una cámara de vídeo que permita acreditar la identidad del estudiante que se está examinando y que enfoque su pantalla, su teclado y el puesto de examen; unido al empleo de aplicaciones que impidan la conexión entre el dispositivo desde el que se realiza el examen y otros (tipo e-proctoring).

El mayor problema es que un caso práctico abarca menos materia, como regla general, que una serie de preguntas cortas sobre diversas partes del programa.

\section{E) Ejercicios de composición}

Al igual que en el caso anterior, esta modalidad de exámenes (en los que se plantea la redacción de un tema de cierta extensión, que no coincide exactamente con ningún epígrafe o subepígrafe concreto de las unidades del programa, pero que está relacionado con varios de dichos epígrafes y unidades), en modo telemático, tampoco plantea especiales problemas de veracidad de los resultados, al ser igualmente difícil su fraude porque no favorece copiar y pegar textos extraídos de otros archivos.
En modo telemático, los ejercicios de composición son un tipo de exámenes que no suelen plantear especiales problemas de veracidad de los resultados y permiten demostrar los conocimientos de los estudiantes sobre diversos temas del programa, así como sus habilidades intelectuales

Como hemos indicado anteriormente, los ejercicios de composición son muy interesantes porque permiten demostrar los conocimientos que tienen los estudiantes sobre diversos temas del programa, así como sus habilidades intelectuales.

Ahora bien, como en el caso anterior, requieren controlar que el examen no sea realizado por terceras personas, para lo cual resulta necesario el uso de una cámara de vídeo con la que poder acreditar la identidad del estudiante que se está examinando y que enfoque su pantalla, el teclado y el puesto de examen; unido al uso de aplicaciones que impidan la conexión entre el dispositivo desde el que se realiza el examen y otros (tipo e-proctoring).

Pero su principal problema es que se trata de un tipo de exámenes aptos para estudiantes de nivel medio-alto, ya que a los alumnos de nivel medio y medio-bajo les resultan difíciles. 


\section{F) Examen de preguntas cortas, en positivo, sobre ideas esenciales y de relación de ideas}

Estos exámenes se caracterizan por preguntas cortas (tipo «ensayo", según se denomina en la plataforma Moodle), en positivo, sobre ideas esenciales y de relación de ideas, planteadas en términos distintos a la exposición ordinaria de los epígrafes y subepígrafes,

El examen de preguntas cortas, en positivo, sobre ideas esenciales y de relación de ideas es el tipo óptimo para los exámenes telemáticos que es, desde mi punto de vista, el tipo óptimo para los exámenes telemáticos. Por un lado, porque sus resultados suelen ser veraces, ya que no admiten fácilmente copiar y pegar archivos, lo que suele evitar resultados fraudulentos, especialmente si además se refuerzan con los mecanismos técnicos impeditivos a los que nos venimos refiriendo. $Y$, por otro, porque este tipo de exámenes fomentan el estudio de las diversas cuestiones y apartados del programa por captación de ideas esenciales y de modo relacional.

El único inconveniente es que, para los alumnos de nivel medio, pueden resultar bastante más difíciles que el tipo de preguntas cortas por subepígrafes o párrafos de un epígrafe, aunque no tan difíciles como los ejercicios de composición; si bien, desde el prisma del aprendizaje basado en la captación de ideas medulares de los distintos temas y en la interrelación de las mismas, es el tipo de examen más propicio, a mi juicio.

No obstante, para impedir el uso de vías fraudulentas, esta modalidad de exámenes debe ser controlada a través de cámaras que enfoquen la pantalla del dispositivo del estudiante que se está examinando, su teclado y su puesto de examen, así como, en lo posible, usar aplicaciones que impidan la apertura de archivos en el dispositivo de realización del examen y la conexión de este con otros dispositivos externos (tipo e-proctoring).

Puede ejecutarse, igualmente, de modo oral, a través de videoconferencia, y, en tal caso, se disminuye, aún más, la posibilidad de fraude.

Es necesaria su grabación ${ }^{16}$, como medio de prueba, fundamentalmente con vistas a solicitudes de revisión y posibles reclamaciones de la calificación otorgada. Pero, como en el caso de los exámenes orales de preguntas cortas de apartados concretos o subepígrafes, también es aconsejable cambiar de preguntas de un estudiante a otro. Esto supone que el docente debe dedicar muchísimo tiempo y esfuerzo en la preparación y realización del examen, y, por tanto, parece impracticable a partir de determinado número de estudiantes y de preguntas.

16 Véase, también, la guía de la Universidad Carlos III de Madrid (2020, p. 8). 


\section{La especial problemática jurídica del uso de cámara de control en la realización de los exámenes telemáticos y de la grabación de dichos exámenes, así como de los demás medios de vigilancia informática (e-proctoring)}

\subsection{Problemática jurídica del uso de cámara de control en la rea- lización de los exámenes telemáticos}

En cuanto a la mera utilización de cámaras, el Informe de la Agencia Española de Protección de Datos 36/2020 considera que es proporcional el visionado con cámara para identificarse, etc., pues hay que tener en cuenta que el Real Decreto 1791/2010 exige, en su artículo 25.7, la acreditación de la identidad, a juicio del evaluador, en cualquier momento.
En cuanto a la mera utilización de cámaras, el Informe de la Agencia Española de Protección de Datos 36/2020 considera que es proporcional el visionado con cámara para identificarse, etc.

La Sentencia 186/2000, del 10 de julio, del Tribunal Constitucional (FJ 5. ${ }^{\circ}$ ) -y las que esta cita- destacó que, en casos con riesgo de comisión de actos fraudulentos, entra en funcionamiento el principio de proporcionalidad, que exige ponderar el derecho a la intimidad y el derecho a la protección de datos con los otros derechos que tienen que sopesarse en la balanza, resaltando que ningún derecho es absoluto y que, cuando uno entra en tensión con otros derechos o con otros bienes jurídicos o valores en conflicto, tiene que ser ponderado con los mismos.

Por su parte, la Sentencia 78/2020, de 13 de julio, del Juzgado de lo ContenciosoAdministrativo núm. 3 de Córdoba, que ha enjuiciado el recurso interpuesto por un estudiante, debido al cambio de los sistemas de evaluación presenciales por los telemáticos, como consecuencia de la pandemia de la COVID-19, con previsión de utilización de cámaras y de grabación, en su caso, primero, desestimó la solicitud de medidas cautelares solicitadas por el estudiante y, finalmente, el recurso.

En relación con el derecho a la intimidad y a la inviolabilidad del domicilio, dice que no tiene por qué ser vulnerado, dado que «es el propio alumno el que decide la ubicación de la cámara, incluso el fondo real o virtual de que pueda dotarse a la escena, con tal de que se le vea a él durante la realización de la prueba, y a los solos efectos de controlar que la hace él mismo, y no otra persona, y sin ayuda».

Ello ha sido ratificado por la Sentencia 2382/2020, de 26 de noviembre, del Tribunal Superior de Justicia de Andalucía, Sala de lo Contencioso-Administrativo de Sevilla (rec. apelación 1447/2020), que desestima el recurso de apelación frente a la anterior sentencia de instancia. 


\subsection{Problemática jurídica de la grabación de exámenes tele- máticos}

Antes del inicio de la pandemia provocada por la COVID-19, se admitía, sin objeciones, la grabación de los exámenes orales. Así, el Informe 10601/2019 de la Agencia Española de Protección de Datos lo considera, para tal tipo de exámenes, proporcional y que se encuentra amparado en el Reglamento (UE) 2016/679, del Parlamento Europeo y del Consejo, de protección de datos.

Tal informe no lo considera proporcional en otras pruebas no orales presenciales, pero ello no debería llevarnos a su exclusión en los exámenes no orales telemáticos.

Por un lado, porque tal informe no alude a otro método de igual eficacia para conseguir el mismo fin, que es lo que requeriría el principio de proporcionalidad para excluirlo. Y es que dicho principio conlleva el denominado «juicio de necesidad», que exige que no exista una medida menos restrictiva, a igualdad de eficacia; de modo que, si no se indica ni acredita cuál es esa medida menos restrictiva pero igual de eficaz, la medida en cuestión se muestra como necesaria y, por ende, proporcional.

Y, por otro lado, porque «a dicha proporcionalidad de la grabación con cámara apunta la jurisprudencia del Tribunal Constitucional», en la Sentencia 186/2000 (FJ 7. ${ }^{\circ}$, párr. primero) y en la Sentencia 39/2016, después, pues admite tal tipo de grabación "cuando hay indicios de que, de otro modo, se van a cometer actos fraudulentos" (en el caso enjuiciado, hurto de dinero por empleados de cajas de establecimientos comerciales); «indicios que existen claramente en los exámenes académicos, desde el momento que podemos encontrar múltiples sentencias de órganos judiciales contencioso-administrativos que han enjuiciado sanciones impuestas por tal tipo de fraude», incluso en exámenes presenciales, «y las han ratificado» 17 .

Y la citada Sentencia 78/2020, de 13 de julio, del Juzgado de lo Contencioso-Administrativo núm. 3 de Córdoba, ratificada por la también citada Sentencia 2382/2020, de 26 de noviembre, del Tribunal Superior de Justicia de Andalucía, ha mantenido que «la posibilidad de grabar la prueba no es inexorable» y que, "en las pruebas escritas, se desaconseja la grabación y solo se permite si el profesor lo considera imprescindible, concurriendo causa justificada debido a la tipología de la prueba de evaluación».

Por tanto, dicha Sentencia «admite la grabación cuando el profesor lo considere absolutamente necesario, siempre que exista razón justificativa en función del medio de evaluación utilizado».

17 Véase Tardío (2020, pp. 139 y ss.). 
Y la citada jurisprudencia constitucional «avala la grabación, cuando hay indicios de que, de otro modo, se van a cometer actos fraudulentos»; indicios que son claros, en el caso de los exámenes telemáticos, habida cuenta de la evidencia de la existencia de múltiples supuestos en los que los estudiantes han incurrido, en sus respuestas, en literalidad manifiesta respecto de archivos de texto de los apuntes o del material didáctico facilitado por el profesorado para el estudio o, incluso, de archivos existentes en internet.

Porque, además, no solo está en juego el derecho a la propia imagen, a la intimidad, a la inviolabilidad del domicilio o a la protección de datos del estudiante tomado como referencia, sino también el cumplimiento de otras normas y la garantía de derechos del resto del alumnado.

Así, hay que considerar, en el otro lado de la balanza, la grave conducta fraudulenta por parte del estudiante infractor hacia la sociedad y el incumplimiento del deber de estudio del alumno (recogido en el art. 46.1 de la LOU y en el art. 13.2 a) del Real Decreto 1791/2010, de 30 de diciembre, por el que se aprueba el estatuto del estudiante universitario); la inobservancia del deber de «abstenerse de la utilización o cooperación en procedimientos fraudulentos en las pruebas de evaluación, en los trabajos que se realicen o en documentos oficiales de la universidad» (del art. 13.2 d) del mismo real decreto); y el respeto del estudiante a las normas de convivencia (que, junto con el respeto a otros derechos o bienes de naturaleza constitucional, fue un argumento destacado por el Auto 382/1996 del Tribunal Constitucional para justificar que no se había lesionado el derecho fundamental a la educación del art. 27 de la Constitución española [CE], nada menos que ante la expulsión de un centro docente, en la enseñanza obligatoria).

Y, por otra parte, también puede producirse la lesión del derecho de los demás estudiantes a la igualdad de oportunidades y no discriminación en el ejercicio de sus derechos académicos (recogido en el art. 46.2 b) de la LOU) y del derecho a la evaluación del rendimiento académico con criterios públicos y objetivos (que está recogido en el art. 25.1 del Real Decreto 1791/2010 y, claramente, excluye la evaluación con fraude).

Tales derechos constituyen claramente una manifestación del derecho fundamental de igualdad del artículo 14 de la CE, en conexión con el principio de objetividad en la actuación administrativa del artículo 103.1 de la CE y con los principios de mérito y capacidad. Pues, en relación con estos últimos principios, hay que tener en cuenta que, aunque la CE alude a ellos, al referirse al acceso a la función pública, y la propia LOU los evoca para el acceso a la universidad (art. 42.3), lógicamente deben informar las calificaciones de las correspondientes asignaturas, una vez que se ha accedido a tal institución ${ }^{18}$.

18 Sobre la incidencia de tales deberes de los estudiantes y de los derechos de los demás alumnos que no utilizan mecanismos fraudulentos, a los que se les están generando desventajas comparativas y lesionando tales derechos, puede verse Tardío (2020, pp. 90, 113 y 141). 


\subsection{Tratamiento de los datos personales}

Porque, además, debe rememorarse, en relación con el derecho fundamental a la protección de datos, el artículo 6.1 del Reglamento (UE) 2016/679, que recoge la «licitud del tratamiento» (y por ello su no vulneración) cuando «es necesario para el cumplimiento de una misión realizada en interés público o en el ejercicio de poderes públicos conferidos al responsable del tratamiento»19. Pues no debe olvidarse que la educación superior está calificada como «servicio público» en el artículo 1.1 de la LOU.

La Sentencia de la Sala de lo Contencioso-Administrativo de la Audiencia Nacional de 26 de septiembre de $2013^{20}$ ya recordó la aplicación del precepto de similar contenido del anterior texto normativo de la Unión Europea (art. 7 e) de Directiva 95/46/CE, de 24 de octubre). En un supuesto de enjuiciamiento de si la actuación de un director de colegio de enseñanzas no universitarias había sido lesiva del derecho a la protección de datos de un estudiante, invocó que el tratamiento de los datos (para verificar y probar que se había cometido una infracción disciplinaria grave) fue lícito, entre otros motivos, porque era, en dicho caso, «necesario para el cumplimiento de una misión de interés público» y que «la actividad educativa no solo puede calificarse de misión de interés público, sino, más aún, de verdadero "servicio público" [...] conforme al artículo 108.3 de la Ley orgánica 2/2006, [de 3 de mayo, de educación]» (pues se refería a un nivel educativo no universitario).

Y ¿puede alguien discutir que la prevención, verificación y prueba del fraude en la evaluación del rendimiento académico, en la que, además, su vertiente calificadora tiene efectos públicos, no sea una exigencia del interés público del servicio público de la educación superior? Creo que no.

Como también debe considerarse el apartado f) del citado artículo 6.1 del Reglamento (UE) 2016/679 que dice que el tratamiento será lícito «si es necesario para la satisfacción de intereses legítimos perseguidos [...] por un tercero, siempre que sobre dichos intereses no prevalezcan los intereses o los derechos y libertades fundamentales del interesado que requieran la protección de datos personales».

La Sentencia de la Sección Primera de la Sala de lo Contencioso-Administrativo de la Audiencia Nacional de 26 de abril de $2012^{21}$, en relación con un procedimiento selectivo, declaró que «[...] no es exigible el consentimiento de aquellas personas que participen en un procedimiento de concurrencia competitiva para el tratamiento de las calificaciones obtenidas en dicho procedimiento y ello como garantía y exigencia de los demás participantes para asegurar la limpieza e imparcialidad del procedimiento en el que concurren [...]». Y añadió

\footnotetext{
${ }^{19}$ Así lo invoca la guía de la Universidad Carlos III de Madrid (2020, p. 5).

20 Véase Aranzadi: JUR\2013\317703 (FJ 4. ${ }^{\circ}$.

21 ROJ: SAN 2131/2012 (FJ 2. ${ }^{\circ}$ ).
} 
que «es cierto que la Ley orgánica 15/1999 no recoge expresamente exenciones o excepciones al régimen de tratamiento de datos personales en ella contenida con fundamento en las garantías de transparencia de los procesos competitivos, por lo que será preciso ponderar los intereses en conflicto para poder determinar cuál de ellos debe prevalecer. Efectuada dicha ponderación, y valorando las circunstancias que aquí concurren, es claro para este tribunal que debe prevalecer en este caso la garantía de publicidad y transparencia del proceso competitivo sobre el derecho a la protección de datos [...]».

Por ello, el Informe 0178/2014, de la Agencia Española de Protección de Datos, invocó la argumentación anterior de la citada sentencia de 2012 de la Audiencia Nacional, para llegar a la misma conclusión en materia de la publicación de las calificaciones académicas de los estudiantes universitarios.

Y a igual conclusión, aunque con otros matices, se ha llegado en el Informe 10601/2019, de la misma agencia, aun teniendo en cuenta el nuevo Reglamento (UE) 2016/679 del Parlamento Europeo y del Consejo, de 27 de abril de 2016, relativo a la protección de las personas físicas en lo que respecta al tratamiento de datos personales y a la libre circulación de estos datos, y la Ley orgánica 3/2018, de 5 de diciembre, de protección de datos personales y garantía de los derechos digitales.

Se han considerado prevalentes los derechos de los otros posibles afectados por desventajas comparativas (en aquellos casos, el derecho de acceso a los documentos, con invocación en el art. 105 b) de la CE) y los principios de publicidad y de transparencia (entonces, art. 3.4 de la Ley 30/1992, y hoy, art. 3.1 c) de la Ley 40/2015) sobre el derecho a la protección de datos.

Pues se recuerda, en dichos informes, que las calificaciones de un estudiante, aunque en principio no derivan de procedimientos de concurrencia competitiva, son de interés personal para los demás alumnos, por la incidencia en las matrículas de honor concedidas, en las becas $^{22}$ o en los premios extraordinarios ${ }^{23}$.

\subsection{Reglamentos de necesidad}

Por último, debe tenerse en cuenta que, en situaciones de «estado de necesidad», como las que ha generado la pandemia de la COVID-19, pueden estar justificadas medidas que no lo estarían en situaciones de normalidad, es decir, medidas que pueden llegar a contradecir lo establecido en las normas con rango de ley y que solo serían aplicables durante

\footnotetext{
22 Véase el final de los epígrafes III y VI del Informe 0178/2014.

23 Véase el final del epígrafe I del Informe 0036/2019.
} 
el periodo temporal en el que inciden tales circunstancias, perdiendo su legitimidad fuera de las mismas, salvo que tuvieran otra cobertura normativa más allá de tales situaciones.

Por eso, se admiten los denominados «reglamentos de necesidad», que pueden llegar a excepcionar la aplicación de normas con rango de ley cuando se dan esas situaciones de estado de necesidad formalmente declaradas (como en los estados de alarma, excepción y sitio [arts. 55 y 116 de la CE] o no declaradas formalmente (como las que pueden se adoptadas por los alcaldes, en virtud del art. $21.1 \mathrm{~m}$ ) de la Ley 7/1985, de 2 de abril, reguladora de las bases del régimen local; o a adoptar por las autoridades sanitarias, en el art. 26 de la Ley 14/1986, de 25 de abril, general de sanidad, entre otros) ${ }^{24}$.

Pero ¿qué ocurre si no están previstos expresamente en ámbitos como el universitario, donde no hay un precepto específico que diga que pueden aprobarse normas exigidas por el estado de necesidad?

Pues a mi entender, en dichos casos, opera el «principio general del derecho de necesidad» al que alude el profesor Álvarez (2005, p. 1.653), quien sostiene que dicho principio está reconocido en las Sentencias 33/1992 y 329/1993 del Tribunal Constitucional.

Desde mi punto de vista, es un principio implícito ${ }^{25}$ en los preceptos (normas-regla) constitucionales que regulan los decretos-leyes (art. 86) o los estados de alarma, excepción y sitio (arts. 55 y 116) y en las normas con rango de ley citadas (art. $21.1 \mathrm{~m}$ ) de la Ley 7/1985 y art. 26 de la Ley 14/1986, entre otras).

Tal principio legitimaría, en situaciones de estado de necesidad, a los órganos competentes en cada caso, para aprobar normas que resuelvan las cuestiones exigidas por la garantía

24 Así lo explican García de Enterría y Fernández (2017, ps. 248-249), aunque también se está invocando lo establecido en la Ley orgánica 3/1986, de 14 de abril, de medidas especiales en materia de salud pública: («[...] medidas oportunas para el control de los enfermos, de las personas que estén o hayan estado en contacto con los mismos y del medioambiente inmediato, así como las que se consideren necesarias en caso de riesgo de carácter transmisible» [art. 3], «[...] cuando así lo exijan razones sanitarias de urgencia o necesidad” [art. 1]).

E, igualmente, se está evocando la Ley 33/2011, de 4 de octubre, general de salud pública, cuyo artículo 54.1 dispone que, «sin perjuicio de las medidas previstas en la Ley orgánica 3/1986, de 14 de abril, de medidas especiales en materia de salud pública, con carácter excepcional, y cuando así lo requieran motivos de extraordinaria gravedad o urgencia, la Administración general del Estado y las de las comunidades autónomas y ciudades de Ceuta y Melilla, en el ámbito de sus respectivas competencias, podrán adoptar cuantas medidas sean necesarias para asegurar el cumplimiento de la ley». Lo que completa con las medidas que enumera en su artículo 54.2.

25 Sobre la figura de los principios generales del derecho implícitos, reconocidos tanto por la jurisprudencia del Tribunal Constitucional como por la del Tribunal Supremo, puede verse su análisis en el libro de Tardío (2011, pp. 91 y ss.). 
del interés general en tales situaciones, aunque excepcionen el derecho ordinario, incluido el aprobado con rango de ley, si es necesario, durante el periodo que dure tal situación.

Y con tales normas, los órganos universitarios citados pueden regular la enseñanza telemática y sus exámenes durante la situación de estado de necesidad, con medidas adecuadas para garantizar el interés general y el derecho de los estudiantes (incluidos los de aquellos a los que el alumno que incide en fraude deja en desventaja comparativa) que cumplan con el principio de proporcionalidad.

\subsection{Conclusiones}

Podemos finalizar este trabajo exponiendo que, al menos, mientras dure la pandemia y sus distintas etapas, la introducción, por acuerdos de órganos de las universidades españolas, de mecanismos no solo de videovigilancia de los exámenes telemáticos, sino también de la grabación y conservación de esta, del periodo estricto que dure el plazo de revisión y reclamación y de las herramientas propias de la denominada e-proctoring no deben ser considerados lesivos de los derechos anteriormente citados, los cuales se han venido invocando para impedir el uso de tales medios antifraude.
La introducción, por acuerdos de órganos de las universidades españolas, de mecanismos no solo de videovigilancia de los exámenes telemáticos, sino también de la grabación y conservación de esta, del periodo estricto que dure el plazo de revisión y reclamación y de las herramientas propias de la denominada e-proctoring no deben ser considerados lesivos de los derechos citados en este trabajo

En relación con las citadas herramientas, son especialmente útiles las que impiden al estudiante la apertura de otras pestañas o pantallas y la conexión con internet y otros dispositivos informáticos, así como las que incorporan el control del sonido de la zona del puesto de examen o que la vista no se aparte reiteradamente de la pantalla donde este se verifica ${ }^{26}$.

Las que utilizan el reconocimiento facial y datos biométricos, que parecen ser las más problemáticas desde el punto de vista de la protección de datos, no son siempre estrictamente necesarias y podrían relegarse exclusivamente para los supuestos en los que resultasen imprescindibles.

${ }^{26}$ Así se indica en la guía de la Universidad Carlos III de Madrid (2020): «[...] medidas como los sistemas de bloqueo del entorno de trabajo del estudiante durante la evaluación (LockDown Browser) no implican ningún tratamiento de datos personales, sino que son una manifestación de la potestad de organización y buen orden en el aula» (p. 6). 


\section{Referencias bibliográficas}

Álvarez García, V. (2005). Necesidad. En S. Muñoz Machado (Dir.), Diccionario de derecho administrativo (Tomo II). lustel.

Bordas Alsina, M. ${ }^{a}$ I. y Cabrera Rodríguez, F. Á. (2001). Estrategias de evaluación de los aprendizajes centrados en el proceso. Revista Española de Pedagogía, 218, 25-48.

Castillo Arredondo, S. y Cabrerizo Diago, J. (2009). Evaluación educativa de aprendizajes y competencias. Pearson.

Comenius, I. A. (1630). Didáctica magna (trad. española publicada en ebook por ePubLibre, con prólogo de Gabriel de la Mora).

Estepa Alonso, R. M. ${ }^{a}$, Vozmediano Torres, J. M. y Estepa Alonso, A. J. (2020). Reflexiones sobre la gestión de riesgos en la evaluación telemática dentro del contexto de la ETSI de la Universidad de Sevilla. Departamento de Ingeniería Telemática de la Universidad de Sevilla.

García de Enterría E. y Fernández Rodríguez, T. R. (2017). Curso de derecho administrativo (Tomo I, 18. ${ }^{a}$ ed.). Thomson-Civitas.

González, M., Marco, E. y Medina, T. (2020). Informe de iniciativas y herramientas de evaluación online universitaria en el contexto del COVID-19. Ministerio de Universidades; Gabinete del Ministro.

Kramer, L. (1999). Derecho ambiental y Tratado de la Comunidad Europea (3. ${ }^{a}$ ed.). Marcial Pons.

López Gómez, E. (2016). La evaluación en el proceso de enseñanza-aprendizaje. En E. López Gómez (Coord.), M. L. Cacheiro, C. Camilli y J. L. Fuentes, Didáctica general y formación del profesorado. UNIR.
Martínez Martínez, R., Arenas Ramiro, M. y Gumbau Mezquita, J. P. (2020). Informe sobre el impacto normativo de los procedimientos de evaluación online: protección de datos y garantía de los derechos de las y los estudiantes. CRUE Universidades Españolas; Secretarías Generales.

Moreu Carbonell, E. (2003). El examen en el nuevo sistema educativo español: régimen jurídico de los exámenes académicos. Comares.

Servadio, A., Berardi, A., Tramontano, M., Tofani, M., Álvaro, R., Farsetti, P. y Galeoto, G. (2020). Description of the method used by Tor Vergata University of Rome for the electronic final exam of the degree course in physiotherapy. International Conference e-Learning 2020 (pp. 165-169).

Tardío Pato, J. A. (1986). El control jurisdiccional de los concursos de méritos, las oposiciones y los exámenes académicos. Civitas, SA.

Tardío Pato, J. A. (1996). La función calificadora de los alumnos universitarios y su control jurisdiccional. Revista de Administración Pública, 139, 373-417.

Tardío Pato, J. A. (2011). Los principios generales del derecho: su aplicación efectiva como normas jurídicas. Bosch.

Tardío Pato, J. A. (2020). Las sanciones disciplinarias a los alumnos universitarios y no universitarios, en centros públicos y privados. Ministerio de Educación y Formación Profesional.

Universidad Carlos III de Madrid. (2020). Guía de pruebas de evaluación en tiempos de docencia online por emergencia. 